\title{
PARAMETERIZATION INVARIANCE AND THE RESOLUTION OF THE UNITARY GAUGE PUZZLE
}

\author{
P.F. Kelly \\ Center for Theoretical Physics, Laboratory for Nuclear Science \\ Massachusetts Institute of Technology \\ Cambridge, MA 02139 USA \\ R. Kobes \\ Lab. de Phys. Théor. ENSLAPP, BP 110 \\ F-74941 Annecy-le-Vieux FRANCE \\ and \\ Winnipeg Institute for Theoretical Physics and Physics Department \\ University of Winnipeg \\ Winnipeg, MB R3B 2E9 CANADA \\ G. Kunstatter \\ Winnipeg Institute for Theoretical Physics and Physics Department \\ University of Winnipeg \\ Winnipeg, MB R3B 2E9 CANADA
}

\begin{abstract}
We examine the calculation of the critical temperature $T_{c}$ for the restoration of a spontaneously broken symmetry. Motivated by a set of recently developed gauge dependence identities, we give a gauge and parameterization independent definition of this temperature in terms of the physical mass of the scalar particle as determined by the propagator pole. As an explicit illustration, we consider the Abelian Higgs model in the unitary gauge, where the usual definition of the critical temperature based upon the effective potential leads to an erroneous result. We show how the gauge invariant definition reproduces the correct result as found in the "renormalizable" parameterizations.

$$
\begin{array}{r}
\text { MIT }- \text { CTP }-2299 \\
\text { WIN }-93-09 \\
\text { ENSLAPP }- \text { A }-470 / 94 \\
\text { NSF }- \text { ITP }-94-62
\end{array}
$$
\end{abstract}




\section{INTRODUCTION}

That there are models in which symmetries, spontaneously broken at low (zero) temperature, are restored at high (finite) temperature was conjectured [1], and explicitly confirmed $[2,3]$ a number of years ago. Quantitative investigations of the Abelian Higgs model have exposed what has become known as the Unitary Gauge Puzzle [2-6]. This puzzle arose when attempts were made to estimate the transition or critical temperature for the restoration of the symmetry; in the unitary gauge, a different value for the critical temperature was obtained as compared to those obtained in certain other gauges. This difference has, in the past, been attributed to pathologies of the unitary gauge and the related question of the validity of the loop expansion. The purpose of this paper is to provide a detailed explanation for the discrepancy as well as a proof that identical results for the critical temperature can in principle be obtained in the unitary gauge and other gauges provided that one uses the correct definition of the critical temperature and that it is evaluated in a self-consistent expansion.

In order to set the stage for the detailed presentation of this puzzle in the next section, we first briefly review the usual approach to calculating the critical temperature using the effective potential [2]. This function, being defined for constant field configurations, enables the survey of a large class of translationally invariant expectation values of the field. The ground state of the system is identified with the global minimum of the effective potential, and spontaneous symmetry breaking is signalled by a non-zero vacuum expectation value (VEV) of the field. Under the assumption that the transition to the symmetric phase takes place in a continuous (i.e., second-order) fashion, the relevant VEVs must go to zero smoothly as the temperature is raised to its critical value. Thus, the "origin" must be a local maximum of the effective potential in the broken phase, and the condition signaling the onset of the second-order phase transition is that the origin be both an extremum and an inflection point of the effective potential.

For concreteness, we consider a complex scalar field parameterized in terms of a real "Higgs" mode $\phi_{H}$ and a real "Goldstone" boson $\phi_{G}$. The order-parameter, whose vanishing signifies the onset of the phase transition, is provisionally identified with the effective thermal mass-squared of the Higgs field:

$$
m_{H \text { eff }}^{2}=\left.\frac{\partial^{2} V\left(\phi_{H}, \phi_{G}, \beta\right)}{\partial^{2} \phi_{H}}\right|_{\substack{\phi_{H}=\phi_{H \text { min }} \\ \phi_{G}=0}} .
$$

The second derivative is evaluated at the value of the VEVs which gives rise to the minimum of the effective potential. Thus, as the temperature increases from below the critical 
temperature, both $m_{H \text { eff }}^{2}$ and the VEV decrease towards zero. Above the critical temperature, $m_{H \text { eff }}^{2}$ is positive and the VEV is zero. The critical temperature is thus defined to be that temperature which causes $m_{H \text { eff }}^{2}$ to vanish at zero VEV:

$$
0=\left.\frac{\partial^{2} V\left(\phi_{H}, \phi_{G}, \beta_{c}\right)}{\partial^{2} \phi_{H}}\right|_{\substack{\phi_{H}=0 \\ \phi_{G}=0}}
$$

This definition of the critical temperature, however, is problematic for gauge theories, since the effective potential, calculated from off-shell Green functions at zero external four-momentum, is gauge dependent $[2,7]$ and parameterization dependent [8]. Several approaches may be taken to deal with these dependences. One is to consider only objects which are manifestly gauge independent (in this context, the Vilkovisky-DeWitt effective action [9] is particularly suitable), as the critical temperature defined by such means must be gauge and parameterization invariant [10]. Alternatively, one can look for signals for symmetry breaking in terms of expectation values of gauge invariant composite operators [11]. Another approach is to try to extract gauge invariant information from gauge dependent objects like the usual effective potential [7]. In this regard the "Ward-like" Nielsen identities are useful in determining the gauge and parameterization dependence of quantities. For example, the Nielson identities have been exploited to show that symmetry breaking and restoration are gauge invariant phenomena [12]. Recently, they have been generalized to obtain a set of algebraic identities which describe the gauge dependence of all $n$-point functions derivable from the effective action [13]. As we shall demonstrate, these identities guarantee that the same result for the critical temperature in any gauge or parameterization can be obtained order-by-order in a self-consistent expansion.

This paper is organized as follows. In Section 2, the unitary gauge puzzle shall be briefly reviewed and previous analyses of it discussed. We shall emphasize results found by Ueda [4] and by Arnold, Braaten and Vokos (ABV) [6]. Section 3 discusses the gauge dependence identities and how they provide a formal resolution to the unitary gauge puzzle to all loop-orders. A two-loop calculation by ABV is then shown to corroborate these formal arguments. Further support for this resolution is presented in Section 4, where we consider a complex $\Phi^{4}$ theory which also suffers from a "unitary gauge puzzle" for different parameterizations of the complex field [8]. Using the definition of the critical temperature discussed in Section 3, we demonstrate that the correct result is obtained in a large class of parameterizations. This class includes a parameterization corresponding to the one which gave rise to the original unitary gauge puzzle. Section 5 contains a brief restatement of our conclusions. 


\section{THE UNITARY GAUGE PUZZLE}

Consider a complex scalar field $\Phi$ with a renormalizable self-interaction, $m^{2}|\Phi|^{2}+$ $(\lambda / 6)|\Phi|^{4}$, respecting the inherent $U(1)$ global symmetry of the system. By gauging this symmetry, a model of scalar electrodynamics is obtained. The model with $m^{2}=-\mu^{2}$ is the standard Abelian Higgs model with spontaneous symmetry breaking:

$$
\mathcal{L}=\left(\mathcal{D}_{\mu} \Phi\right)^{\dagger}\left(\mathcal{D}^{\mu} \Phi\right)+\mu^{2}|\Phi|^{2}-\frac{\lambda}{3 !}\left(|\Phi|^{2}\right)^{2}-\frac{1}{4} F_{\mu \nu} F^{\mu \nu}+\mathcal{L}_{\substack{\text { GAXIXE } \\ \text { FIING }}}
$$

The gauge-covariant derivative couples the gauge field to the scalar with strength $e: \mathcal{D}_{\mu} \Phi=$ $\left[\partial_{\mu}+i e A_{\mu}\right] \Phi$, and the $U(1)$ field strength tensor is $F_{\mu \nu}=\partial_{\mu} A_{\nu}-\partial_{\nu} A_{\mu}$. The gauge fixing term has been left unspecified at this point. Before one is able to progress further, one must choose a parameterization for the complex field. There are two distinct options which shall be discussed in turn.

\section{1) Cartesian parameterization}

The complex field $\Phi$ may be decomposed into its real and imaginary parts:

$$
\Phi=\frac{1}{\sqrt{2}}\left[{ }^{R} \phi+i^{I} \phi\right] .
$$

Spontaneous symmetry breaking produces an asymmetric vacuum state about which the true quantum fields $\left\{R_{f}, I_{f}\right\}$ fluctuate. Without loss of generality, the entire zero temperature VEV of the scalar field (i.e., $\rho_{0} / \sqrt{2}$ ) may be assigned to the real component:

$$
R_{\phi}=\rho_{0}+R_{f}, \quad I_{\phi}=0+{ }^{I_{f}} .
$$

The vector field $A_{\mu}$ fluctuates about a zero VEV. Note that the functional Jacobian corresponding to the change of field variables from $\left\{\Phi, \Phi^{*}\right\} \rightarrow\left\{{ }_{f}, I_{f}\right\}$ is unity and thus the path integral measure is trivial.

Feynman rules based upon the Lagrangian written in terms of these shifted fields may be derived in a straightforward manner. In order to do this, a gauge fixing term must be specified, but the results are independent of this choice. The order-parameter as defined by the curvature of the effective potential may also be expressed as the self-energy of the Higgs field $R_{f}$ evaluated at zero external four-momentum. At one-loop:

$$
m_{R \text { eff }}^{2}=m_{R}^{2}+\left.\Pi_{\substack{\text { TaCTAL } \\ \text { vacuum }}}^{(R R)}\left(P^{2}\right)\right|_{P=(0, \overrightarrow{0})}+\left.\prod_{\substack{\text { TorAL } \\ \text { thermal }}}^{(R R)}\left(P^{2}\right)\right|_{P=(0, \overrightarrow{0})} .
$$

The $m_{R}^{2}$ term is the bare tree-level mass-squared which is combined with the divergent one-loop vacuum self-energy and renormalized in the usual way. Explicit calculation of 
the finite temperature contribution leads to, at leading-order, and in the high temperature limit,

$$
m_{\text {Reff }}^{2}=2 \mu^{2}-\frac{\lambda}{9} \frac{1}{\beta^{2}}-\frac{1}{2} e^{2} \frac{1}{\beta^{2}}+O\left(\beta^{-1}\right) .
$$

The terms which arise from the scalar sector have coupling strength $\lambda$, while those from the gauge-field loops couple with $e^{2}$. It is assumed that $\lambda$ and $e^{2}$ are of the same order. Setting the expression for the order-parameter (2.5) to zero results in an equation which leads to the (correct) estimate of the critical temperature [2]:

$$
0=2 \mu^{2}-\left[\frac{\lambda}{9}+\frac{1}{2} e^{2}\right] \frac{1}{\beta_{c}^{2}}+O\left(\beta_{c}^{-1}\right) .
$$

\section{2) Polar parameterization}

An alternative approach to this analysis is to use the magnitude and phase representation of the complex field:

$$
\Phi=\frac{1}{\sqrt{2}} \rho e^{i \theta / \rho_{0}},
$$

where $\langle\Phi\rangle=\rho_{0} / \sqrt{2}$ is the VEV of the scalar field at zero temperature. Furthermore, all explicit dependence upon the Goldstone mode $\theta$ is eliminated by choosing the "unitary" gauge:

$$
B_{\mu}=A_{\mu}+\frac{1}{g \rho_{0}} \partial_{\mu} \theta \text {. }
$$

Spontaneous symmetry breaking affects the modulus field alone:

$$
\rho=\rho_{0}+\rho_{f}
$$

Thus, only the "physical" degrees of freedom are present: a massive scalar Higgs boson ${ }^{\circ}$, and a massive vector meson $B_{\mu}$.

Feynman rules for the Lagrangian expressed in terms of these fields may then be derived. Note that the gauge for the vector field has been specified by (2.8). The model in this form has lost its property of being manifestly renormalizable due to the large momentum behaviour of the vector meson propagator. The transformation of field variables gives rise to a non-trivial functional Jacobian in the path-integral measure, which may be incorporated into the Lagrangean in the usual manner,

$$
\mathcal{L}_{\text {ghost }}=-\bar{c}\left(1+\frac{\rho f}{\rho_{0}}\right) c,
$$

where $\{\bar{c}, c\}$ are scalar fields obeying Fermi-Dirac statistics. Notwithstanding these complications, the calculation of the one-loop self-energy of the Higgs field can be performed 
in a straightforward manner. At zero external four-momentum, one finds in the high temperature limit the following equation for the order parameter:

$$
m_{\rho \mathrm{eff}}^{2}=2 \mu^{2}-\frac{\lambda}{12} \frac{1}{\beta^{2}}-\frac{1}{2} e^{2} \frac{1}{\beta^{2}}+O\left(\beta^{-1}\right)
$$

Setting this expression equal to zero produces an equation for the critical temperature,

$$
0=2 \mu^{2}-\left[\frac{\lambda}{12}+\frac{1}{2} e^{2}\right] \frac{1}{\beta_{c}^{2}}+O\left(\beta_{c}^{-1}\right) .
$$

which does not agree with the equation (2.6) obtained in the Cartesian parameterization. This disagreement constitutes the unitary gauge puzzle. It is noteworthy that the contribution from the gauge field sector is the same in both instances. This suggests that the unitary gauge puzzle arises from the scalar sector of the Abelian Higgs model, rather than the gauge field sector.

\section{3) Previous Analyses of the Unitary Gauge Puzzle}

It has been suggested that the apparent non-renormalizability of the Abelian Higgs model in the unitary gauge and the concommitant question of the validity of the loopexpansion is to blame for the unitary gauge puzzle. However, Ueda [4] observed that by using an improved order-parameter [the second-derivative of the effective potential plus " $\Pi\left(P^{2}=-m^{2}\right)-\Pi\left(P^{2}=0\right)$ "] it was possible to obtain the same result for the transition temperature in the unitary gauge as in other gauges. As we shall see in the next section, this corresponds to the pole-definition of the Higgs mass. In the Cartesian parameterization, the one-loop self-energy is independent of the external four-momentum and thus this additional term vanishes. However, in the unitary gauge the momentumdependent vertices give rise to a non-zero contribution which exactly accounts for the difference between (2.6) and (2.12).

More recently, ABV re-analysed the unitary gauge puzzle and determined two general criteria which must be met for a calculation of the critical temperature to yield a result which is meaningful. The first criterion is that the order-parameter itself must be the physical, gauge-invariant mass-squared of the Higgs field as defined by the location of the

propagator pole. The second criterion is that the perturbative expansion and the ultimate evaluation of physical quantities under investigation must be self-consistently undertaken. They performed a calculation of the critical temperature in the unitary gauge in powers of $T / T_{c}$ valid for low temperatures, by consistently extracting the leading temperature dependence in a loop-expansion. In this way, they argued that it was possible to obtain agreement between the Cartesian gauge and the unitary gauge values of the critical 
temperature, provided that certain unsuppressed terms of the form $\left(T / T_{c}\right)^{2 n}$ for $n \geq 2$ which arose in the unitary gauge calculation appeared with vanishing coefficients. Through careful analysis they were able to show explicitly that the $O\left(T^{4}\right)$ terms did indeed cancel among themselves up to two-loop-order. They were, however, unable to prove or verify that this property persisted for all higher-orders. Moreover, in the ABV method of analysis, it seemed that cancellations were required between one- and two-loop contributions to the self-energy.

In the next section, a general framework justifying Ueda's approach shall be presented. Furthermore, in this context, the "miraculous" cancellations found by ABV demonstrate the consistency of the loop-expansion in the unitary gauge to two-loop-order.

\section{GAUGE DEPENDENCE IDENTITIES}

The off-shell effective action and the effective potential depend explicitly on the gauge and the field parameterization in which they are calculated. However, this dependence is controlled by a set of generalized Ward identities that can be derived from the definition of the effective action, or its Legendre transform, the generating functional for connected diagrams. A complete discussion of these identities, and further references, can be found in [13]. The generalized Ward identities yield an equation that determines the gauge dependence of the two-point function $D_{i j}^{-1}$ in a field theoretic model. In the absence of sources, the equation reads:

$$
\Delta D_{i j}^{-1}=D_{i k}^{-1} \Delta X_{j}^{k}+D_{j k}^{-1} \Delta X_{i}^{k}
$$

where we employ a condensed notation in which $\{i, j, k, \ldots\}$ refer to all discrete field indices as well as to continuous spacetime coordinates. In the above, $\Delta X_{j}^{i}$ is a quantity that can be calculated in perturbation theory. At this stage, however, (3.1) is exact as it stands. The crucial feature to note is that the change in the two-point function is proportional to the inverse-propagator itself.

For the scalar mode in the spontaneously broken Abelian Higgs model at finite temperature, the identities acquire a particularly simple form in momentum space:

$$
\Delta\left(p^{2}-m^{2}-\Pi\left(p^{2} ; \rho^{2}, T\right)\right)=\left(p^{2}-m^{2}-\Pi\left(p^{2} ; \rho^{2}, T\right)\right) \Delta X .
$$

In the above equation, $m^{2}=2 \mu^{2}$ is the renormalized tree-level mass of the Higgs field and a Minkowski metric is used. The self-energy $\Pi$ can in principle depend on both $p$, the external four-momentum, and on $\rho$, the VEV of the scalar field. Clearly, (3.2) implies that the propagator pole-position as determined by

$$
p^{2}=m^{2}+\Pi\left(p^{2} ; \rho^{2}, T\right)
$$


is both gauge and parameterization invariant, provided of course that the corresponding $\Delta X$ is well behaved there.

It must be emphasized that (3.2) is exact. Thus, the temperature dependent mass as defined by the propagator pole, and hence the critical temperature, can in principle be computed in either unitary or renormalizable gauges to yield the same result. This is not the case for the curvature of the effective potential, i.e., the inverse-propagator at zero external four-momentum, which is gauge dependent, in general. An exception to this arises when the self-energy is independent of momentum, as happens in the renormalizable gauges to one-loop-order. However, when the self-energy does depend on momentum, it is necessary to use the pole-position definition of the temperature dependent mass in order to obtain the correct result for the critical temperature.

There is a line of argument that one might choose to follow in the hope of expediting the analysis which, unfortunately, leads to erroneous conclusions. At the precise value of the critical temperature, the physical situation demands that the VEV vanish and that the propagator pole occur at $p^{2}=0$. Thus, one is tempted to specialize (3.3), and solve

$$
0=m^{2}+\Pi\left(0 ; 0, T_{c}\right),
$$

for the critical temperature. If (3.4) were the case, then it would always be possible to obtain the correct, gauge invariant, critical temperature from the effective potential. However, this argument presumes an exact knowledge of the self-energy, whereas in practice it is necessary to evaluate it perturbatively. We shall now show that the critical temperature obtained from the pole-position definition of the mass is gauge and parameterization invariant order-by-order in any self-consistent expansion (including the loop-expansion). This is not, however, true for calculations based on (3.4).

There are two distinct and yet equivalent ways in which the dependence on the VEV can be consistently accounted for in the self-energy. The first is to treat the VEV as a parameter to be solved for perturbatively from the condition that the effective potential attain its minimum there. In this case, the self-energy is computed from the usual set of 1PI diagrams. The second approach is to include "tadpole" diagrams in the calculation of the self-energy. In this case one demands that the tree-level tadpole vanish, thus obtaining the relation given below in (3.6). The parameter $\rho$ appearing in (3.3) et.seq., is set to its tree-level value to enable consistent iterative solution for the pole-position. Higher-order tadpole loop contributions are responsible for the evolution of the VEV, and their inclusion in the self-energy takes these effects into account. In this section, we adopt the former approach because that is the framework employed by ABV, making it easier for us to utilize their results at two-loops in the unitary gauge (c.f. Eq.(3.6) of Ref.[6]). However, 
in the next section, the latter method is chosen, where only the self-energy needs to be calculated (with extra tadpole diagrams) rather than both the self-energy and the effective potential.

One ordinarily employs a perturbative expansion that can be represented schematically as follows:

$$
\begin{aligned}
& \Pi=l \Pi_{(1)}+l^{2} \Pi_{(2)}+\ldots \\
& \rho^{2}=\rho_{(0)}^{2}+l \rho_{(1)}^{2}+l^{2} \rho_{(2)}^{2}+\ldots
\end{aligned}
$$

Note that the self-energy starts at the first-order in the expansion, since we assume that the zeroth-order action is the classical action ${ }^{1}$. Hence, the lowest-order scalar VEV must solve the classical field equations. For the Abelian Higgs model this leads to

$$
\bar{\rho}_{(0)}^{2}=3 m^{2} / \lambda=6 \mu^{2} / \lambda .
$$

We also assume that renormalization (to absorb all divergences into the parameters of the theory) has been carried out consistently. In particular, the temperature dependent terms in the perturbative expansions considered here are all finite.

In $(3.5 a, b), l$ may be any parameter. It may be a combination of coupling constants, or a formal loop-counting parameter, such as $\hbar$. It is vital that all of the contributions to any given order in the expansion parameter be properly taken into account. If this is done, then the pole-position, as determined by (3.3) is gauge fixing and parameterization independent order-by-order in $l$. Equation (3.3) must be solved with care. The only self-consistent solution takes the form of a Taylor expansion in the arbitrary parameter $l$ :

$$
\begin{aligned}
& \bar{p}^{2}=\bar{p}_{(0)}^{2}+l \bar{p}_{(1)}^{2}+l^{2} \bar{p}_{(2)}^{2}+\ldots \\
& \bar{\rho}^{2}=\bar{\rho}_{(0)}^{2}+l \bar{\rho}_{(1)}^{2}+l^{2} \bar{\rho}_{(2)}^{2}+\ldots
\end{aligned}
$$

where

$$
\begin{aligned}
& \bar{p}_{(0)}^{2}=m^{2}, \\
& \bar{p}_{(1)}^{2}=\left.\Pi_{(1)}\left(p^{2} ; \rho^{2}, T\right)\right|_{\begin{array}{l}
p^{2}=m^{2} \\
\rho^{2}=\bar{\rho}_{(0)}^{2}
\end{array}}, \\
& \bar{p}_{(2)}^{2}=\left.\Pi_{(2)}\left(p^{2} ; \rho^{2}, T\right)\right|_{\begin{array}{l}
p^{2}=m^{2} \\
\rho^{2}=\bar{\rho}_{(0)}^{2}
\end{array}}+\left.\bar{p}_{(1)}^{2} \frac{\partial \Pi_{(1)}}{\partial p^{2}}\right|_{\substack{p^{2}=m^{2} \\
\rho^{2}=\bar{\rho}_{(0)}^{2}}}+\left.\bar{\rho}_{(1)}^{2} \frac{\partial \Pi_{(1)}}{\partial \rho^{2}}\right|_{\substack{p^{2}=m^{2} \\
\rho^{2}=\bar{\rho}_{(0)}^{2}}},
\end{aligned}
$$

1 In field theories that require resummation due to infrared divergences this is not quite true, but this subtlety has no relevance to the present discussion. 
and so on. The successive terms in the expansion of $(3.7 b)$ are determined by analysis of the effective potential. The identities (3.2) guarantee algebraically that $\bar{p}_{(1)}^{2}, \bar{p}_{(2)}^{2}$, etc., are each separately gauge and parameterization independent. Note that the correct evaluation of $\bar{p}_{(1)}^{2}$ has required setting the self-energy on its bare mass-shell $p^{2}=m^{2}$, as opposed to $p^{2}=$ 0 . In renormalizable gauges, since the self-energy is independent of the external momentum to this order, this distinction is moot. In unitary gauge, the distinction is crucial, and accounts for the discrepancy between the correct value for the critical temperature and that obtained from an expansion based on (3.4). Indeed, the gauge dependence identity for the two-point function, expanded to one-loop (first-order in the perturbative parameter) yields

$$
\Delta \Pi_{(1)}\left(0, \rho_{(0)}^{2}, T\right)=-m^{2} \Delta X_{(1)}
$$

which is not equal to zero in general. However, $\Delta X_{(1)}=0$ for renormalizable gauges and thus the method based on analysis of the effective potential works at one-loop in this class of gauges. In unitary gauge the self-energy is momentum dependent, and hence gauge and parameterization dependent as well, except on-shell. For cases of this type, the poleposition and associated self-consistent expansion outlined above must be used in order to get an invariant estimate for the particle mass and hence the critical temperature.

In the Abelian Higgs model, it was first shown by Ueda [4] that, in the high temperature limit,

$$
\Pi_{(1)}=-2\left(\lambda / 2+3 e^{2}\right) \frac{T^{2}}{12}-\frac{p^{2}}{\rho_{0}^{2}} \frac{T^{2}}{12}
$$

in the unitary gauge. Thus the one-loop correction to the pole-position as obtained from $(3.8 b)$,

$$
\bar{p}_{(1)}^{2}=-2\left(\frac{\lambda}{2}+\frac{\lambda}{6}+3 e^{2}\right) \frac{T^{2}}{12},
$$

coincides precisely with the value obtained in renormalizable gauges. Fortunately, the elegant unitary gauge calculations of $\mathrm{ABV}$ are available to verify that the identities work at two-loops as well. The leading-order, high temperature contributions to the two-loop self-energy sum to

$$
\Pi_{(2)}=\frac{\lambda}{2 \rho_{0}^{2}}\left(\frac{T^{2}}{12}\right)^{2}-\frac{5}{2} \frac{p^{2}}{\rho_{0}^{4}}\left(\frac{T^{2}}{12}\right)^{2}+O\left(T^{2}\right)
$$

while the shift in the scalar VEV is determined to be

$$
\bar{\rho}_{(1)}^{2}=-\frac{6}{\lambda}\left(\lambda / 2+3 e^{2}\right) \frac{T^{2}}{12},
$$


and thus it is straightforward to verify that

$$
\bar{p}_{(2)}^{2}=O\left(T^{2}\right)
$$

The terms of order $T^{2}$ which contribute to the second-order correction in (3.12), appear with higher powers of the coupling constants, viz., $\left\{\lambda^{2}, e^{4}, \lambda e^{2}\right\}$, and so they are of higherorder than are the terms contributing to $\bar{p}_{(1)}^{2}$ in $(3.10 b)$ above.

Note that throughout this section we treat $l$ as a loop-counting parameter. As pointed out by $\mathrm{ABV}$, in unitary gauge, the loop-expansion corresponds to an expansion in $T^{2}$. In principle, therefore, the two-loop contribution could generate a large correction $O\left(T^{4}\right)$ to the one-loop result $O\left(T^{2}\right)$. The fact that this does not occur is a direct consequence of the gauge dependence identities, which guarantee that the same result to any order must be obtained in unitary gauge as in renormalizable gauges. In renormalizable gauges it is manifestly clear that the net $O\left(T^{4}\right)$ contribution must vanish at two-loop-order. Note that the expression $(3.8 c)$ for $\bar{p}_{(2)}^{2}$ involves both $\Pi_{(2)}$ and derivatives of $\Pi_{(1)}$. ABV made this observation and interpreted it as a cancellation between one-loop and two-loop terms. They were led to conclude that generic calculations in unitary gauge would require contributions from all higher-loops in order to agree with results obtained in renormalizable gauges. In light of the gauge dependence identities, their analysis shows rather that one must keep all relevant contributions to the required order in the perturbative (in this case, the loopcounting) parameter.

\section{THE COMPLEX $\Phi^{4}$ MODEL}

In order to see more clearly the issue of parameterization dependence in the perturbative computation of the critical temperature, we consider an interacting complex scalar field suffering spontaneous symmetry breaking whose global $U(1)$ symmetry is not gauged. The Lagrange density is

$$
\mathcal{L}=\left(\partial_{\mu} \Phi\right)^{\dagger}\left(\partial^{\mu} \Phi\right)+\mu^{2}|\Phi|^{2}-\frac{\lambda}{3 !}\left(|\Phi|^{2}\right)^{2}
$$

We introduce a one-parameter family of parameterizations in terms of two real fields $\left\{{ }^{1} \phi,{ }^{2} \phi\right\}$ and a real parameter $\epsilon$ :

$$
\begin{aligned}
& \Phi=\frac{1}{\sqrt{2}}\left[{ }^{R_{\phi}}+i^{I} \phi\right], \\
& R_{\phi}=(1-\epsilon){ }^{1} \phi+\epsilon{ }^{1} \phi \cos \left({ }^{2} \phi / \rho_{0}\right), \\
&{ } \phi=(1-\epsilon)^{2} \phi+\epsilon{ }^{1} \phi \sin \left({ }^{2} \phi / \rho_{0}\right) .
\end{aligned}
$$

Inspection of $(4.2-4)$ reveals that the Cartesian parameterization of Section 2.1 is obtained for $\epsilon=0$, while the polar parameterization corresponding to the unitary gauge in Section 
2.2 results when $\epsilon=1$. For $0<\epsilon<1$, an "interpolating" parameterization ensues. Both fields $\left\{\frac{1}{\phi},{ }^{2} \phi\right\}$ are chosen to have the same canonical dimension as $\Phi$; the scale associated with the angular field is identified here with the zero temperature VEV. This restriction shall be relaxed in Section 4.1 .

The Lagrangian (4.1) may be expressed in terms of $\left\{{ }^{1} \phi,{ }^{2} \phi\right\}$ by substitution of $(4.2-4)$. The zero temperature VEV of the scalar field may be chosen to be concentrated entirely in the $1 \phi$ mode,

$$
{ }^{1} \phi=\rho_{0}+1 f, \quad{ }^{2}=0+{ }^{2} f .
$$

Substitution of (4.5) into the $\left\{\frac{1}{\phi},{ }^{2} \phi\right\}$-Lagrangian corresponds to the usual shift of the fields by their VEVs. The presence of the trigonometric functions in $(4.3,4)$ leads to the appearance of vertices to all orders in $\left\{1_{f} f, 2\right\}$. However, only those with up to four legs are required to construct the self-energies to one-loop-order. The Lagrangian is

$$
\mathcal{L}=\mathcal{L}_{\text {constant }+ \text { linear }}+\mathcal{L}_{\text {quadratic }}+\mathcal{L}_{\text {cubic }}+\mathcal{L}_{\text {quartic }}+\mathcal{L}_{\text {higher-order }}
$$

where

$$
\begin{aligned}
& \mathcal{L}_{\text {constant }+ \text { linear }}=\frac{1}{2} \mu^{2} \rho_{0}^{2}-\frac{\lambda}{4 !} \rho_{0}^{4}-m_{2}^{2} \rho_{0} y, \\
& \mathcal{L}_{\text {quadratic }}=-\frac{1}{2} 1 f\left[\partial_{\mu} \partial^{\mu}+m_{1}^{2}\right] 1 f-\frac{1}{2} 2^{2}\left[\partial_{\mu} \partial^{\mu}+(1-\epsilon) m_{2}^{2}\right]^{2} f, \\
& \mathcal{L}_{\text {cubic }}=-\frac{1}{3 !} \lambda \rho_{0} f^{3}-\frac{1}{2} \frac{\lambda \rho_{0}}{3}(1-\epsilon) f^{2} f^{2}+\frac{1}{2} \frac{2}{\rho_{0}} \epsilon^{1} f\left(\partial_{\mu}^{2} f\right)\left(\partial^{\mu}{ }^{2} f\right), \\
& \mathcal{L}_{\text {quartic }}=-\frac{1}{4 !} \lambda \mathcal{f}^{4}-\frac{1}{4} \lambda\left[\frac{1}{3}(1-\epsilon)+\left(\frac{2 \mu^{2}}{\lambda \rho_{0}^{2}}-\frac{1}{3}\right) \epsilon(1-\epsilon)\right] f^{2} 2 f^{2} \\
& +\frac{1}{4} \lambda \frac{2}{\lambda \rho_{0}^{2}} \epsilon^{2} f^{2}\left(\partial_{\mu}^{2} f\right)\left(\partial^{\mu 2} f\right) \\
& -\frac{1}{4} \lambda \frac{2}{\lambda \rho_{0}^{2}} \epsilon(1-\epsilon)\left(\partial_{\mu} f\right)\left(\partial^{\mu} 1 f\right)^{2} f^{2} \\
& -\frac{1}{4} \lambda \frac{4}{\lambda \rho_{0}^{2}} \epsilon(1-\epsilon)^{1} f\left(\partial_{\mu} f\right)^{2} f\left(\partial^{\mu} 2 f\right) \\
& -\frac{1}{4 !} \lambda\left[(1-\epsilon)^{2}+\left(\frac{3 \mu^{2}}{\lambda \rho_{0}^{2}}-\frac{1}{2}\right) \epsilon(1-\epsilon)\right]{ }^{2} f^{4} \\
& -\frac{1}{4} \lambda \frac{2}{\lambda \rho_{0}^{2}} \epsilon(1-\epsilon)^{2} f^{2}\left(\partial_{\mu}^{2} f\right)\left(\partial^{\mu} f\right)
\end{aligned}
$$

and

$$
m_{1}^{2}=-\mu^{2}+\frac{\lambda}{2} \rho_{0}^{2}, \quad m_{2}^{2}=-\mu^{2}+\frac{\lambda}{6} \rho_{0}^{2} .
$$


Propagators:

$$
\begin{array}{ccc} 
& \Delta(k)= & \frac{1}{k^{2}+m_{1}^{2}} \\
\ldots & 2 \Delta(k)= & \frac{1}{k^{2}} \\
\ldots \ldots \ldots \ldots . . . & 1
\end{array}
$$

Cubic Vertices:

$$
\begin{aligned}
& -g_{1}\left(q_{1}, q_{2}, q_{3}\right) \delta\left(\sum q\right) \quad g_{1}=\lambda \rho_{0} \\
& \square^{\prime} \quad-g_{12}\left(q_{1} ; q_{2}, q_{3}\right) \delta\left(\sum q\right) \quad g_{12}=\frac{\lambda \rho_{0}}{3}(1-\epsilon)-\frac{2}{\rho_{0}} \epsilon q_{2} \cdot q_{3} \\
& -g_{1 \bar{c} c}\left(q_{1} ; q_{2}, q_{3}\right) \delta\left(\sum q\right) \quad g_{1 \bar{c} c}=\frac{1}{\rho_{0}} \epsilon
\end{aligned}
$$

Quartic Vertices:

$$
\begin{aligned}
& \chi \quad-\lambda_{1}\left(q_{1}, q_{2}, q_{3}, q_{4}\right) \delta\left(\sum q\right) \quad \lambda_{1}=\lambda \\
& y^{\prime} \quad-\lambda_{12}\left(q_{1}, q_{2} ; q_{3}, q_{4}\right) \delta\left(\sum q\right) \quad \lambda_{12}=\lambda\left[\begin{array}{c}
\frac{1}{3}(1-\epsilon)+\frac{2}{\lambda \rho_{0}^{2}} \epsilon(1-\epsilon) q_{1} \cdot q_{2} \\
+\frac{1}{\lambda \rho_{0}^{2}} \epsilon(1-\epsilon)\left[\begin{array}{c}
q_{1} \cdot q_{3}+q_{1} \cdot q_{4} \\
+q_{2} \cdot q_{3}+q_{2} \cdot q_{4}
\end{array}\right] \\
-\frac{2}{\lambda \rho_{0}^{2}} \epsilon^{2} q_{3} \cdot q_{4}
\end{array}\right] \\
& \text { ‘, } \\
& -\lambda_{2 \bar{c} c}\left(q_{1}, q_{2} ; q_{3}, q_{4}\right) \delta\left(\sum q\right) \quad \lambda_{2 \bar{c} c}=-\frac{2}{\rho_{0}^{2}} \epsilon(1-\epsilon)
\end{aligned}
$$

Table 1: Feynman rules for the interacting complex scalar model expressed in terms of the interpolating parameterization. The solid, dashed and dotted lines denote Higgs (typeone), Goldstone (type-two) and ghost field propagators, respectively. The Goldstone boson is massless as a consequence of the tree-level stability condition. For the vertices, momenta are positive incoming and are enumerated in an anticlockwise fashion beginning from the upper left corner. Each of the vertices contains an energy(frequency)-momentum conserving delta-function.

The presence of the derivative interactions obscures the manifest renormalizability of the model. In addition, the field transformations from $\left\{\Phi, \Phi^{*}\right\} \rightarrow\left\{{ }^{1} f,{ }^{2} f\right\}$ produce a non-trivial Jacobian term in the functional measure, which can be represented through 
Grassman-valued ghost fields as

$$
\mathcal{L}_{\text {ghost }}=-\bar{c}\left(1+\frac{1}{\rho_{0}} \epsilon \bar{f}-\frac{1}{\rho_{0}^{2}} \epsilon(1-\epsilon)^{2} f^{2}+\ldots\right) c .
$$

The terms which have been omitted (represented by ellipses) are of higher-order in the fields and hence will not contribute to the one-loop self-energies.

Feynman rules for this parameterization at finite temperature in the imaginary time formalism are collected in Table 1. Appropriate to this formalism, in this section we work with Euclidean four-momentum $P_{\mu}$; the Minkowski mass-shell relation $p^{2}=m^{2}$ of the previous section translates here into $P^{2}=-m^{2}$. As discussed in Section 3, the viewpoint which shall be adopted here is to incorporate the effects of the evolution of the VEV by inclusion of tadpole graphs in the computation of the self-energies. By adhering to this prescription, only the self-energy needs to be computed. The parametric value of the VEV in (3.3) is set by the classical Lagrangian, see (4.10), in order that the perturbative series be well-formed. Discarding the linear term in the Lagrangian (4.6) corresponds to dispensing with the tree-level tadpole diagram, and leads to the tree-level stability condition c.f., $(3.6)$ :

$$
m_{2}^{2}=0 \quad \Longleftrightarrow \quad m_{1}^{2}=\frac{\lambda}{3} \rho_{0}^{2}=2 \mu^{2} \quad \Longleftrightarrow \quad \rho_{0}^{2}=\frac{6 \mu^{2}}{\lambda}
$$

The collection of diagrams contributing to the self-energy of the Higgs mode $\left(I_{f}\right)$ is assembled in Figure 1, with the corresponding expressions found in Table 2.

a1)

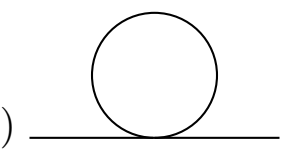

b1)

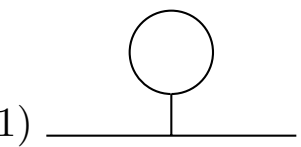

c1)

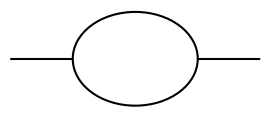

a2)

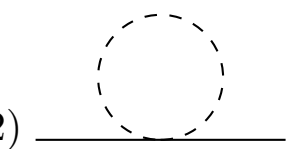

b2)

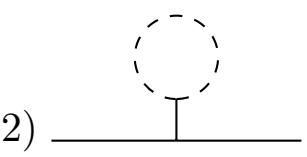

c2)

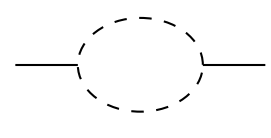

bg)

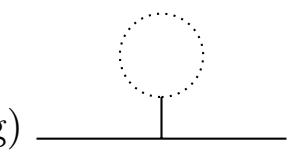

cg)

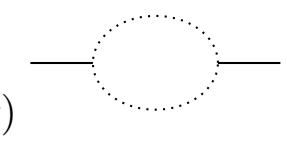

Figure 1: The self-energy of the Higgs field to one-loop-order as expressed in terms of the interpolating parameterization.

The effective mass-squared of the Goldstone boson shall also be explicitly calculated so as to provide a consistency check on the application of the method and its results. The set of self-energy diagrams for the Goldstone mode $(2 f)$ is found in Figure 2, and their expressions are listed in Table 3. 
$\underline{\text { Diagram }}$

\begin{tabular}{|c|c|c|}
\hline a1 & $\int d k-\frac{\lambda}{2}{ }^{1} \Delta(k)$ & $T^{2}$ \\
\hline $\mathrm{a} 2$ & $\int d k\left\{-\frac{1}{\rho_{0}^{2}} \epsilon^{2}-\frac{\lambda}{2}\left(\frac{1}{3}(1-\epsilon)-\frac{2 P^{2}}{\lambda \rho_{0}^{2}} \epsilon(1-\epsilon)\right)^{2} \Delta(k)\right\}$ & $T^{4}+T^{2}$ \\
\hline b1 & $\int d k \frac{\lambda}{2} \frac{\lambda \rho_{0}^{2}}{m_{1}^{2}}{ }^{2} \Delta(k)$ & $T^{2}$ \\
\hline b2 & $\int d k\left\{\frac{\lambda}{m_{1}^{2}} \epsilon+\frac{\lambda}{2}\left(\frac{\lambda \rho_{0}^{2}}{3 m_{1}^{2}}(1-\epsilon)\right)^{2} \Delta(k)\right\}$ & $T^{4}+T^{2}$ \\
\hline bg & $\int d k-\frac{\lambda}{m_{1}^{2}} \epsilon$ & $T^{4}$ \\
\hline $\mathrm{c} 1$ & $\int d k \frac{\lambda}{2}\left(\lambda \rho_{0}^{2}\right) \Delta(k) \Delta(P-k)$ & $" T^{0} "$ \\
\hline$c 2$ & $\int d k\left\{\begin{array}{cc}\frac{2}{\rho_{0}^{2}} \epsilon^{2} & -\frac{\lambda}{2}\left[\begin{array}{l}\left(\frac{4 P^{2}}{\lambda \rho_{0}^{2}} \epsilon^{2}-\frac{4}{3} \epsilon(1-\epsilon)\right)^{2} \Delta(P-k) \\
-\frac{4}{\lambda \rho_{0}^{2}} \epsilon^{2}(P \cdot k)^{2} \Delta(k)^{2} \Delta(P-k)\end{array}\right. \\
+ \text { lower order terms }\end{array}\right.$ & $T^{4}+T^{2}$ \\
\hline & $\int d k-\frac{1}{\rho_{0}^{2}} \epsilon^{2}$ & $T^{4}$ \\
\hline
\end{tabular}

Table 2: The self-energy contributions to the (11) two-point function at one-loop order which arise in the interacting complex scalar model as expressed in the interpolating parameterization. Here, $\int d k \equiv \frac{1}{\beta} \sum_{n_{k}} \int \frac{d \vec{k}}{(2 \pi)^{3}}$.

a1)

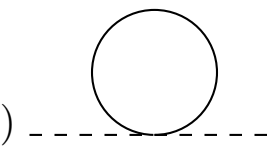

b1)

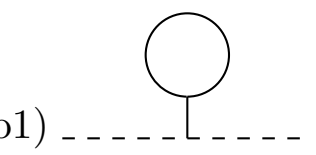

$\mathrm{c} 12)$

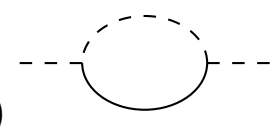

a2)

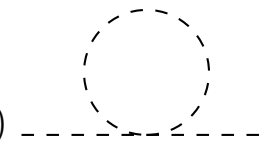

b2) $\ldots . . . .-T^{\prime} \ldots$

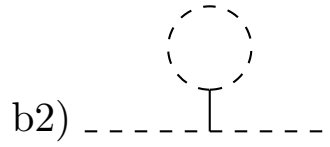

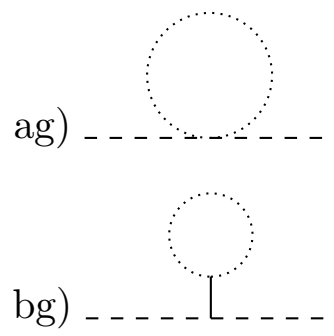

Figure 2. The self-energy to one-loop-order of the Goldstone mode, as expressed in terms of the interpolating parameterization.

In Tables 2 and 3, the leading naive degrees of temperature dependence associated with each diagram are given. We note that the vacuum contributions which are quartically divergent (associated with the $O\left(T^{4}\right)$ pieces) all cancel.

To evaluate each of these self-energies, the field is chosen to be at rest with respect to the heat bath. In the high temperature limit, the following results hold:

$$
\left.\int d k \frac{1}{k^{2}+m^{2}}\right|_{\text {thermal }}=\frac{J(\beta m)}{\beta^{2}}
$$




$$
\begin{aligned}
& \text { Diagram } \quad-\Pi^{(22)}(P) \quad \underline{\text { Naive Order }} \\
& \text { a1 } \int d k\left\{\frac{1}{\rho_{0}^{2}} \epsilon(1-\epsilon)-\frac{\lambda}{2}\left[\begin{array}{c}
\frac{1}{3}(1-\epsilon)+\frac{2 P^{2}}{\lambda \rho_{0}^{2}} \epsilon^{2} \\
+\frac{2 m_{1}^{2}}{\lambda \rho_{0}^{2}} \epsilon(1-\epsilon)
\end{array}\right] \unlhd(k)\right\} \quad T^{4}+T^{2} \\
& \text { a2 } \int d k\left\{\frac{1}{\rho_{0}^{2}} \epsilon(1-\epsilon)-\frac{\lambda}{2}\left[\begin{array}{c}
(1-\epsilon)^{2} \\
-\frac{2 P^{2}}{\lambda \rho_{0}^{2}} \epsilon(1-\epsilon)
\end{array}\right]{ }^{2} \Delta(k)\right\} \quad T^{4}+T^{2} \\
& \text { ag } \int d k-\frac{2}{\rho_{0}^{2}} \epsilon(1-\epsilon) \quad T^{4} \\
& \text { b1 } \int d k \frac{\lambda}{2}\left(\frac{\lambda \rho_{0}^{2}}{3 m_{1}^{2}}(1-\epsilon)+\frac{2 P^{2}}{m_{1}^{2}} \epsilon\right) \Delta(k) \quad T^{2}
\end{aligned}
$$

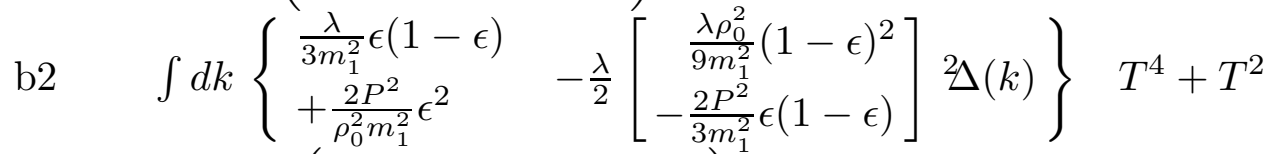

$$
\begin{aligned}
& \text { bg } \int d k-\left(\frac{\lambda}{3 m_{1}^{2}} \epsilon(1-\epsilon)+\frac{2 P^{2}}{\rho_{0}^{2} m_{1}^{2}} \epsilon^{2}\right) \quad T^{4} \\
& \text { c12 } \int d k \lambda \frac{4}{\lambda \rho_{0}^{2}} \epsilon^{2}(P \cdot k)^{2} \Delta(k) 2 \Delta(P-k) \quad T^{2}
\end{aligned}
$$

Table 3: The self-energy contributions to the (22) two-point function at one-loop-order which arise in the interacting complex scalar model as expressed in the interpolating parameterization.

$$
\begin{aligned}
& \left.\int d k \frac{(P \cdot k)^{2}}{\left(k^{2}+m^{2}\right)\left((P-k)^{2}+m^{2}\right)}\right|_{\substack{\text { thermal } \\
\text { at rest }}}=\frac{1}{2} P^{2} \frac{J(\beta m)}{\beta^{2}}+O\left(\beta^{-1}\right), \\
& \left.\int d k \frac{(P \cdot k)^{2}}{\left(k^{2}+m^{2}\right)\left((P-k)^{2}+M^{2}\right)}\right|_{\substack{\text { thermal } \\
\text { at rest }}} \simeq \frac{1}{2} P^{2} \frac{J(\beta m)}{\beta^{2}}+O\left(\beta^{-1}\right),
\end{aligned}
$$

where

$$
J(c)=\frac{1}{2 \pi^{2}} \int_{0}^{\infty} d x \frac{x^{2}}{\sqrt{x^{2}+c^{2}}} \frac{1}{e^{\sqrt{x^{2}+c^{2}}}-1} .
$$

Moreover, in the high temperature limit $\beta m \rightarrow 0$,

$$
J(\beta m)=\frac{1}{12}+O(\beta m) .
$$

This limit, as well as $\beta M \rightarrow 0$, is already assumed in equation (4.11).

The order-parameter for the symmetry restoring phase transition is the effective masssquared of the Higgs field,

$$
m_{1 \text { eff }}^{2}=m_{1}^{2}+\left.\prod_{\substack{\text { TOTAL } \\ \text { thermal }}}^{(11)}\left(P^{2}\right)\right|_{\substack{\text { at rest } \\ \text { hight temp. } \\ \text { on-shell }}} .
$$

Note that renormalization is assumed to have taken place, and $m_{1}^{2}$ represents the physical value of the zero temperature Higgs boson mass-squared. In the high temperature (weak 
coupling) limit, we find

$$
m_{1 \mathrm{eff}}^{2}=2 \mu^{2}-\left.\frac{\lambda}{9 \beta^{2}}\left\{1-\frac{1}{4}\left(1+\frac{P^{2}}{m_{1}^{2}}\right)[\epsilon(2 \epsilon-1)]\right\}\right|_{\text {on-shell }}+O\left(\beta^{-1}\right) .
$$

Thus, on-shell at $P^{2}=-m_{1}^{2}$ the coefficient of the $\epsilon$-dependent term vanishes identically, and the final expression for the order-parameter is

$$
m_{1 \mathrm{eff}}^{2}=2 \mu^{2}-\frac{\lambda}{9 \beta^{2}}+O\left(\beta^{-1}\right)
$$

Equation (4.16) is valid for the entire $\epsilon$-family of parameterizations and yields the correct critical temperature (c.f., $(2.5-6))$ in all cases. This is the central result of our analysis of the interacting complex scalar model and has provided a practical illustration of the gauge-dependence identities.

Note that in the $\epsilon=0$ limit of the general result (4.15) the term in square brackets vanishes, and the correct physical one-loop result for the value of the order-parameter (4.16) then follows independently of the application of the on-shell condition. This is due to the absence of derivative interactions in the Cartesian parameterization. Thus, the method of analysis based upon the effective potential approach as described in Section 2 works in the Cartesian case to one-loop-order. Also note that for the polar case $\epsilon=1$, evaluating the order parameter off-shell at $P_{\mu}=0$ results in the (erroneous) result of the unitary gauge calculated from the effective potential alone:

$$
\left.m_{1 \text { eff }}^{2}\right|_{P=(0, \overrightarrow{0})}=2 \mu^{2}-\frac{\lambda}{12 \beta^{2}}+O\left(\beta^{-1}\right) .
$$

As a test of the validity of the method and the consistency of the calculations, consider the expression for the effective mass-squared of the Goldstone mode:

$$
m_{2 \text { eff }}^{2}=m_{2}^{2}+\prod_{\substack{\text { TOTAL } \\
\text { thermal }}}^{(22)}\left(P^{2}\right) \mid \begin{aligned}
& \begin{array}{l}
\text { at rest } \\
\text { high temp. } \\
\text { on-shell }
\end{array} \\
& .
\end{aligned} .
$$

We expect that the Goldstone boson is massless in the broken phase below $T_{c}$, and indeed collecting all terms in the high temperature limit yields

$$
m_{2 \mathrm{eff}}^{2}=0-\left.\frac{\lambda}{12 \beta^{2}}\left\{\frac{P^{2}}{\lambda \rho_{0}^{2}} \epsilon(5-\epsilon)\right\}\right|_{\text {on-shell }}+O\left(\beta^{-1}\right),
$$

which vanishes at $P^{2}=-m_{2}^{2}=0$, independent of $\epsilon$. Thus, the Goldstone boson remains massless at one-loop to the order at which it is calculated. 


\section{1) The Case of an Arbitrary Scale For the Goldstone Mode}

The calculation described above was undertaken with a parameterization which carried an explicit dependence on the VEV at zero temperature. In this subsection, a twoparameter family of parameterizations will be constructed in which the scale $\rho$ is not identified with the VEV of the field:

$$
\begin{aligned}
\Phi=\frac{1}{\sqrt{2}}\left[{ }_{\phi}+i^{I} \phi\right], \\
R_{\phi}=(1-\epsilon){ }^{1} \phi+\epsilon \phi \cos \left({ }^{2} \phi / \rho\right), \\
I_{\phi}=(1-\epsilon)^{2} \phi+\epsilon{ }^{1} \phi \sin \left({ }^{2} \phi / \rho\right) .
\end{aligned}
$$

This can be written in terms of the $\operatorname{VEV} \rho_{0}$ by introduction of a dimensionless parameter:

$$
\rho=\eta \rho_{0}, \quad 0<\eta<\infty
$$

A frequently occurring combination of $\eta$ and $\epsilon$ shall be denoted by $b$, where

$$
b=\eta+\epsilon-\eta \epsilon=\eta+(1-\eta) \epsilon=\epsilon+(1-\epsilon) \eta .
$$

Although not necessary in this context, it is possible to choose a parameterization for which the standard normalization of the kinetic term for the Goldstone field results; this can be accomplished by using

$$
\begin{aligned}
& R_{\phi}=(1-\epsilon){ }^{1} \phi+\epsilon{ }^{1} \phi \cos \left({ }^{2} \phi / \rho_{0} b\right), \\
& I_{\phi}=\frac{(1-\epsilon) \eta}{b}{ }^{2} \phi+\epsilon{ }^{1} \phi \sin \left({ }^{2} \phi / \rho_{0} b\right),
\end{aligned}
$$

rather than the parameterization of $(4.21-4.22)$ VEVs are then assigned to the fields as

$$
{ }^{1} \phi=\rho_{0}+{ }^{1} f, \quad{ }^{2}=0+{ }^{2} f
$$

The Feynman rules for this parameterization are collected in Table 4. Fortunately, no new vertices are required beyond those listed in Table 1, but the vertex factors are more complicated. As before, the tree-level stability condition (4.10) arises again when elimination of the linear term in the Lagrangian is imposed.

The same diagrams listed in Figure 1 contribute to the self-energy in this more general parameterization. Evaluation of these diagrams in the high-temperature limit leads to the following expression for the order-parameter:

$$
m_{1 \mathrm{eff}}^{2}=2 \mu^{2}-\left.\frac{\lambda}{9 \beta^{2}}\left\{1-\frac{1}{4 b^{2}}\left(1+\frac{P^{2}}{m_{1}^{2}}\right)\left[\epsilon\left(\frac{2 \epsilon}{b}-1\right)\right]\right\}\right|_{\text {on-shell }}+O\left(\beta^{-1}\right) .
$$


Propagators:

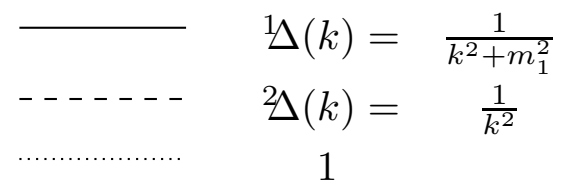

Cubic Vertices:

$$
\begin{array}{lll} 
& -g_{1}\left(q_{1}, q_{2}, q_{3}\right) \delta\left(\sum q\right) & g_{1}=\lambda \rho_{0} \\
\vdots & -g_{12}\left(q_{1} ; q_{2}, q_{3}\right) \delta\left(\sum q\right) & g_{12}=\frac{\lambda \rho_{0}}{3} \frac{b^{2}-\epsilon}{b^{2}}+\frac{\epsilon(1-b)}{\rho_{0} b^{2}}\left[q_{1} \cdot\left(q_{2}+q_{3}\right)\right]-\frac{2 \epsilon}{\rho_{0} b} q_{2} \cdot q_{3} \\
\hdashline & -g_{1 \bar{c} c}\left(q_{1} ; q_{2}, q_{3}\right) \delta\left(\sum q\right) & g_{1 \bar{c} c}=\frac{\epsilon}{\rho_{0} b}
\end{array}
$$

Quartic Vertices:

$$
\begin{aligned}
& \searrow \quad-\lambda_{1}\left(q_{1}, q_{2}, q_{3}, q_{4}\right) \delta\left(\sum q\right) \quad \lambda_{1}=\lambda \\
& y^{\prime} \quad-\lambda_{12}\left(q_{1}, q_{2} ; q_{3}, q_{4}\right) \delta\left(\sum q\right) \quad \lambda_{12}=\lambda\left[\begin{array}{l}
\frac{1}{3} \frac{b^{2}-\epsilon}{b^{2}}-\frac{4 \epsilon(1-b)}{3 b^{2}} \\
+\frac{2 \epsilon(1-\epsilon)}{\lambda \rho_{0}^{2} b^{2}} q_{1} \cdot q_{2} \\
+\frac{\epsilon(1-\epsilon)}{\lambda \rho_{0}^{2} b^{2}}\left[\begin{array}{c}
q_{1} \cdot q_{3}+q_{1} \cdot q_{4} \\
+q_{2} \cdot q_{3}+q_{2} \cdot q_{4}
\end{array}\right] \\
-\frac{2 \epsilon^{2}}{\lambda \rho_{0}^{2} b^{2}} q_{3} \cdot q_{4}
\end{array}\right] \\
& \because x^{\prime}, \quad-\lambda_{2}\left(q_{1}, q_{2}, q_{3}, q_{4}\right) \delta\left(\sum q\right) \quad \lambda_{2}=\lambda\left[\begin{array}{c}
\frac{\left(b^{2}-\epsilon\right)^{2}}{b^{4}} \\
+\frac{2 \epsilon(1-\epsilon) \eta}{\lambda \rho_{0}^{2} b^{4}}\left[\begin{array}{c}
q_{1} \cdot q_{2}+q_{1} \cdot q_{3} \\
+q_{1} \cdot q_{4}+q_{2} \cdot q_{3} \\
+q_{2} \cdot q_{4}+q_{3} \cdot q_{4}
\end{array}\right]
\end{array}\right] \\
& \ddots \quad-\lambda_{2 \bar{c} c}\left(q_{1}, q_{2} ; q_{3}, q_{4}\right) \delta\left(\sum q\right) \quad \lambda_{2 \bar{c} c}=-\frac{\epsilon(1-\epsilon)(1+\eta)}{\rho_{0}^{2} b^{3}}
\end{aligned}
$$

Table 4: Feynman rules for the interacting complex scalar model expressed in terms of the modified interpolating parameterization. The notation and conventions are the same as those used in Table 1.

There are several things to note about this equation. The first is that setting the external four-momentum on-shell, $P^{2}=-m_{1}^{2}$, causes the coefficient of the $\epsilon$ - and $b$-dependent terms to vanish. Thus, the correct physical result is obtained for all values of the parameters. The second feature of (4.28) is that in the limit in which the scale is chosen to equal the VEV, viz., $\eta=1$ and hence $b=1$, then the result reduces to the one obtained 
earlier in (4.15). The third aspect of (4.28) we mention is that its structure guarantees that the correct physical result is obtained to this order both on- and off-shell in the Cartesian parameterization, $\epsilon=0$, as expected. Finally, it is noted that when the polar parameterization, $\epsilon=1$, is chosen, then $b=1$, and the result is manifestly independent of the scale chosen for the angular Goldstone field. This fortuituous independence enabled the unitary gauge puzzle to be more precisely formulated when it was first discovered.

Finally, the Goldstone boson's thermal self-energy corrections may be calculated from the diagrams of Figure 2. The result is:

$$
m_{2 \mathrm{eff}}^{2}=0-\left.\frac{\lambda}{12 \beta^{2}}\left\{P^{2} G(\epsilon, \eta, b)\right\}\right|_{\text {on-shell }}+O\left(\beta^{-1}\right)
$$

where $G(\epsilon, \eta, b)$ is a function of its parameteric arguments. Thus, as was the case in (4.19), evaluating (4.29) on-shell at $P^{2}=0$ shows that the Goldstone boson remains massless at one-loop-order independent of the precise details of the parameterization.

\section{CONCLUSIONS}

We have shown that a complete resolution to the so-called unitary gauge puzzle is obtained by examining the Ward identities that govern the gauge and parameterization dependence of the quantities under consideration. These show directly that the discrepancy between the unitary gauge and renormalizable gauge results for the critical temperature derive not from any intrinsic pathologies of the unitary gauge, but from the momentum dependence of the self-energy. We argued that gauge invariant results for the critical temperature can be obtained order-by-order in any self-consistent perturbative expansion in any gauge, including unitary gauge. This was verified explicitly at the two-loop level to order $T^{4}$ in scalar QED, and at the one-loop level in a family of parameterizations in complex $\phi^{4}$ theory. The latter calculation also stresses the important, but sometimes forgotten, fact that the unitary gauge puzzle is concerned more with parameterization invariance than with gauge invariance, although the two issues are deeply related.

It must be stressed that our analysis does more than re-interpret the two-loop calculations of ABV. First of all, our arguments, admittedly formal, provide an all-order proof that the cancellation observed by $\mathrm{ABV}$ in unitary gauge at the two-loop order occurs to all orders in perturbation theory. Secondly, our analysis provides criteria that can be used to determine whether or not a given perturbative calculation should be expected to yield gauge invariant results. In particular, it is necessary to start at lowest-order from a gauge invariant quantity (such as the pole-position of a physical propagator), and perform a self-consistent expansion. In the present paper the loop-counting parameter was used, but 
of course any convenient parameter may be used (coupling constant, temperature, etc.). It is, however, important to note that although any such self-consistent expansion will guarantee gauge independence order-by-order, the question of accuracy must be addressed independently. The weakness of unitary guage is that this question is obscured due to the presence of non-renormalizable interactions introduced by the non-linear reparameterization. The utility of the gauge dependence identities lies in the fact that they guarantee that the error estimate can be done in any convenient gauge, such as renormalizable gauge, while the actual calculations can be performed in any other gauge, because the same answer will be obtained order-by-order in all gauges. Thus, for example, the accuracy of the leading one-loop result for the critical temperature in renormalizable gauges implies that higher-loop contributions in unitary gauge will also be negligable.

\section{Acknowledgements}

We thank R. Jackiw, E. Braaten and S. Vokos for valuable discussions. This work was supported in part by the Natural Sciences and Engineering Research Council of Canada, the United States Department of Energy under contract \#DE-AC02-76ER03069 and cooperative agreement \#DE-FC02-94ER40818, the National Science Foundation under Grant No. PHY89-04035, and the Centre International des Etudiants et Stagiaires de France. 


\section{REFERENCES}

1. D.A. Kirzhnits and A.D. Linde, Phys. Lett. B42, 471 (1972).

2. L. Dolan and R. Jackiw, Phys. Rev. D9, 3320 (1974).

3. S. Weinberg, Phys. Rev. D9, 3357 (1974).

4. Y. Ueda, Phys. Rev. D23, 1383 (1981).

5. M. Chaichian, E.J. Ferrer and V. de la Incera, Nucl. Phys. B362, 616 (1991).

6. P. Arnold, E. Braaten and S. Vokos, Phys. Rev. D46, 3576 (1992).

7. L. Dolan and R. Jackiw, Phys. Rev. D9, 2904 (1974).

8. G. Kunstatter and H.P. Leivo, Phys. Lett. B183, 75 (1987).

9. G.A. Vilkovisky, in Quantum Theory of Gravity, ed. S.M. Christensen (Adam Hilger, Bristol, 1984); B.S. DeWitt, in, Quantum Field Theory and Quantum Statistics, ed. I.A. Batalin, C.J. Isham and G.A. Vilkovisky, (Adam Hilger, Bristol, 1987).

10 R. Kobes, G. Kunstatter and D.J. Toms, in, TEV Physics, ed. G. Domokos and S. Kovesi-Domokos, (World Scientific, Singapore, 1988).

11. I. Lawrie, J. Phys. A (in press); R. Jackiw and G. Amelino-Camelia, in Banff/CAP Workshop on Thermal Field Theory (World Scientific, Singapore, 1994).

12. N.K. Nielsen, Nucl. Phys. B101, 173 (1975); I.J.R. Aitchison and C.M. Fraser, Ann. of Phys. 156, 1 (1984).

13. R. Kobes, G. Kunstatter and A. Rebhan, Phys. Rev. Lett. 64, 2992 (1990); Nucl. Phys. B355, 1 (1991). 